\title{
The special least squares solutions of the complex matrix equation $(\mathrm{AXB}, \mathrm{CXD})=(\mathrm{E}, \mathrm{F})$
}

\author{
yanzhen Zhang ${ }^{1}$, Shanshan Yang ${ }^{2}$, and Ying $\mathrm{Li}^{1}$ \\ ${ }^{1}$ Liao Cheng University \\ ${ }^{2}$ Beijing Institute of Technology
}

May 6, 2020

\begin{abstract}
In this paper, we study the problem of least square solutions of the complex matrix equation $(\mathrm{AXB}, \mathrm{CXD})=(\mathrm{E}, \mathrm{F})$. First, by using the real representation method of the complex matrix, we prosent the real representation form of the complex matrix equation $(\mathrm{AXB}, \mathrm{CXD})=(\mathrm{E}, \mathrm{F})$. In combination with the special structure of the real representation matrix of the complex matrix, the vec operator of the matrix, the Kronecker product of the matrix and the MP inverse property of the matrix, we obtain the Hermitian least squares solution of the complex matrix equation $(\mathrm{AXB}, \mathrm{CXD})=(\mathrm{E}, \mathrm{F})$, and derive the Hermitian minimum norm least square solution, the real symmetric minimum norm least square solution and the real dissymmetric minimum norm least square solution of the complex matrix equation $(\mathrm{AXB}, \mathrm{CXD})=(\mathrm{E}, \mathrm{F})$. At last, we give the expressions of three minimum norm least squares solutions and their corresponding algorithms, respectively.
\end{abstract}

The special least squares solutions of the complex matrix equation

$$
(\mathbf{A X B}, \mathbf{C X D})=(\mathbf{E}, \mathbf{F})
$$

Yanzhen Zhang ${ }^{1}$, Shanshan Yang ${ }^{2}$, Ying Li ${ }^{1 *}$

1.School of Mathematical Science, Liaocheng University, Liaocheng Shandong 252000

2. School of Mathematics and Statistics, Beijing Institute of Technology, Beijing 100081

Abstract In this paper, we study the problem of least square solutions of the complex matrix equation $(\mathrm{AXB}, \mathrm{CXD})=(\mathbf{E}, \mathbf{F})$. First, by using the real representation method of the complex matrix, we prosent the real representation form of the complex matrix equation $(\mathrm{AXB}, \mathrm{CXD})=(\mathbf{E}, \mathbf{F})$. In combination with the special structure of the real representation matrix of the complex matrix, the vec operator of the matrix, the Kronecker product of the matrix and the MP inverse property of the matrix, we obtain the Hermitian least squares solution of the complex matrix equation $(\mathrm{AXB}, \mathrm{CXD})=(\mathbf{E}, \mathbf{F})$, and derive the Hermitian minimum norm least square solution, the real symmetric minimum norm least square solution and the real dissymmetric minimum norm least square solution of the complex matrix equation $(\mathrm{AXB}, \mathrm{CXD})=(\mathbf{E}, \mathbf{F})$. At last, we give the expressions of three minimum norm least squares solutions and their corresponding algorithms, respectively.

Keywords Matrix equation; Hermitian least squares solution; The real symmetric least square solution; The real opposition least square solution; Real representation matrix.

\section{Introduction}


Matrix equations have a wide range of applications, which the calculation of special least squares solution is always a research hotspot in the field of numerical algebra. The least squares solutions of complex matrix equations have been studied extensively ${ }^{[1]-[11]}$. For example, $[1,2]$ by using the real representation method of the complex matrix, Zhang et al. study the Hermitian minimum norm least square solution of $A X B+C X D=E$ and $(A X B, C X D)=(E, F)$, respectively; [3] by the Kronecker product of the Matrix-Vector and the MP inverse property of the matrix, Wang et al. proposed a direct method to solve the least squares solution of the complex matrix equation $(A X B, C X D)=(E, F)$; [4] Yuan et al. proposed a new method to solve the Hermitian least square solution of the complex matrix equation, and gave the expression and algorithm of the least square solution of the Hermitian least square solution and the Hermitian minimum norm least square solution, et al.

This paper study the problem of least square solutions of complex matrix equations

$(A X B, C X D)=(E, F)$.

In this paper, we use the following notations.

Let $R^{m \times n} C^{m \times n} H C^{n \times n} \mathrm{SR}^{n \times n} \mathrm{ASR}^{n \times n}$ be the sets of $m \times n$ real matrices, be the sets of $m \times n$ real matrices, be the sets of $m \times n$ Hermitian complex matrices, be the sets of $m \times n$ real symmetric matrices, be the sets of $m \times n$ real dissymmetric matrices. $R^{n}$ be the sets of $n$ column vector,

$A^{T} A^{H}$ denote the transpose and the conjugate transpose of matrix $A$, respectively. $A^{+}$denote the MP inverse of matrix $A, I_{n}=\left(e_{1}, e_{2}, \ldots, e_{n}\right)$ denote the identity matrix of order $n,\|A\|$ denote Frobenius norm of complex matrices

For (1), we mainly discuss the following three issues.

Problem 1 Let $A, C \in C^{m \times n}, B, D \in C^{n \times s}, E, F \in C^{m \times s}$, the Hermitian minimum norm least squares solution of (1) denote by $C_{H}$, that is

$$
\mathrm{C}_{H}=\left\{X \mid X \in \mathrm{HC}^{n \times n},\|A X B-E\|+\|C X D-F\|=\min \right\},
$$

make sure $C_{H}$, and solve the Hermitian minimum norm least square solution $\hat{X} \in C_{S}$ satisfying $\|\tilde{X}\|=$ $\min _{X \in C_{H}}\|X\|$.

Problem 2 Let $A, C \in C^{m \times n}, B, D \in C^{n \times s}, E, F \in C^{m \times s}$, the real symmetric minimum norm least squares solution of (1) denote by $C_{S}$, that is

$$
\mathrm{C}_{S}=\left\{X \mid X \in \mathrm{SR}^{n \times n},\|A X B-E\|+\|C X D-F\|=\min \right\}
$$

make sure $C_{S}$, and solve the symmetric minimum norm least square solution $\hat{X} \in C_{S}$ satisfying $\|\hat{X}\|=$ $\min _{X \in C_{s}}\|X\|$.

Problem 3Let $A, C \in C^{m \times n}, B, D \in C^{n \times s}, E, F \in C^{m \times s}$, the real dissymmetric minimum norm least squares solution of (1) denote by $C_{A}$, that is

$$
C_{A}=\left\{X \mid X \in \mathrm{ASR}^{n \times n},\|\mathrm{AXB}-E\|+\|\mathrm{CXD}-F\|=\min \right\},
$$

make sure $C_{A}$, and solve the symmetric minimum norm least square solution $\dot{X} \in C_{A}$ satisfying 足 $\|\dot{X}\|=$ $\min _{X \in C_{A}}\|X\|$.

\section{Preliminaries}

In this paper, we need the following concepts and conclusions: 
Definition $1^{[12]}$ For $A=\left(a_{\mathrm{ij}}\right) \in C^{m \times n}, B=\left(b_{\mathrm{ij}}\right) \in C^{p \times q}$, called the following block matrix

$$
A \otimes B=\left(a_{\mathrm{ij}} B\right) \in C^{m p \times n q}
$$

is the Kronecker product of matrix $A$ and matrix $B$.

Definition $2{ }^{[1]}$ For $A=A_{1}+A_{2} i \in C^{m \times n}$, which $A_{1}, A_{2} \in R^{m \times n}$.

$A^{R}=$

$\left(\begin{array}{cc}A_{1} & a m p ;-A_{2} \\ A_{2} & a m p ; A_{1}\end{array}\right) \in R^{2 m \times 2 n}$,

is called to the real representation matrix of $A$, Let $A_{c}^{R}$ denote the first column block $\left(\begin{array}{l}A_{1} \\ A_{2}\end{array}\right)$ of $A^{R}$.

$A^{R}$ and $A_{c}^{R}$ have some properties as follows:

Lemma $1^{[1]}$ For $A, B \in C^{m \times n}, k \in C$. Then the following properties hold.

(1) $A=B \Leftrightarrow A^{R}=B^{R} ;(2)(A+B)^{R} A^{R}+B^{R} ;(3)(\mathrm{kA})^{\mathrm{R}}=k A^{R}$.

Lemma $2{ }^{[1]}$ For $A, B \in C^{m \times n}, D \in C^{n \times s}, k \in C$. Then the following properties hold.

(1) $A=B \Leftrightarrow A_{c}^{R}=B_{c}^{R} ;(2)(\mathrm{kA})_{c}^{R}=k A_{c}^{R}$,

(3) $(A D)_{c}^{R}=A^{R} D_{c}^{R} ;(4)(A+B)_{c}^{R} A_{c}^{R}+B_{c}^{R}$.

For $A \in C^{m \times n}$, have $\|A\|=\frac{1}{2}\left\|A^{R}\right\|=\left\|A_{c}^{R}\right\|$ and .

The vec operator of real matrix have some properties as follows:

Lemma $3^{[12]}$ For $A \in R^{m \times n}, B \in R^{n \times p}, C \in R^{p \times q}$. Then the following properties hold.

$$
\operatorname{vec}(\mathrm{ABC})=\left(C^{T} \otimes A\right) \operatorname{vec}(B) .
$$

For complex matrix $X=X_{0}+X_{1} i$, the following relationship between $X^{R} X_{C}^{R} X_{0}$ and $X_{1}$ as follows:

Lemma $4^{[1]}$ For $X=X_{0}+X_{1} i \in C^{m \times n}$. Then the following properties hold. (1) $\operatorname{vec}\left(X^{R}\right)=\operatorname{Hvec}\left(X_{C}^{R}\right)$, in which $H=$

$\left(\begin{array}{l}\operatorname{diag}\left(I_{2 n}, \ldots, I_{2 n}\right) \\ \operatorname{diag}\left(Q_{n}, \ldots, Q_{n}\right)\end{array}\right) \in R^{4 m n \times 2 \mathrm{mn}}, Q_{n}=$

$\left(\begin{array}{cc}0 & a m p ;-I_{n} \\ I_{n} & a m p ; 0\end{array}\right)$.

(2) $\operatorname{vec}\left(X_{c}^{R}\right)=K\left(\begin{array}{c}\operatorname{vec} X_{0} \\ \operatorname{vec} X_{1}\end{array}\right)$,

in which $K=$ 


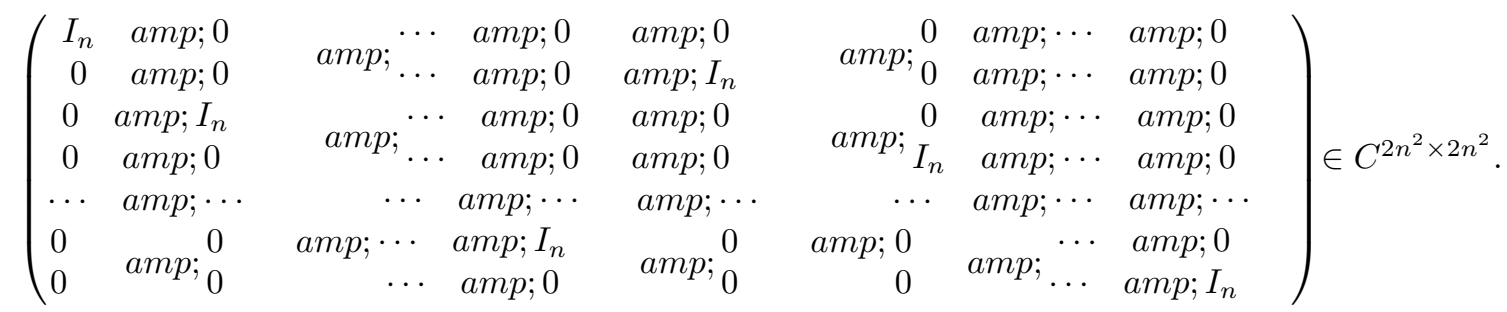

(3) if $X=X_{0}$, have

$\operatorname{vec}\left(X_{c}^{R}\right)=\operatorname{Vvec}\left(X_{0}\right)$,

in which $V=$

$\left(\begin{array}{crrr}I_{n} & a m p ; 0 & a m p ; \cdots & a m p ; 0 \\ 0 & a m p ; 0 & a m p ; \cdots & a m p ; 0 \\ 0 & I_{n} & \cdots & a m p ; 0 \\ 0 & 0 & \cdots & a m p ; 0 \\ \cdots & a m p ; \cdots & a m p ; \cdots & \cdots \\ 0 & 0 & \cdots & a m p ; I_{n} \\ 0 & 0 & \cdots & 0\end{array}\right) \in C^{2 n^{2} \times n^{2}}$

By calculation, give definition and relationship between the independent elements of real (dis)symmetric matrix and vec of its independent elements:

Definition $3^{[1]}$ For $X=\left(x_{\mathrm{ij}}\right) \in \mathrm{SR}^{n \times n}$, let $\alpha_{1}=\left(x_{11}, x_{21}, \ldots x_{n 1}\right), \alpha_{2}=\left(x_{22}, x_{32}\right.$, $\left.\ldots x_{n 2}\right), \ldots, \alpha_{n-1}=\left(x_{(n-1)(n-1)}, x_{n(n-1)}\right), \alpha_{n}=x_{n n}$, the elements of $\alpha_{1}, \alpha_{2}, \ldots, \alpha_{n}$ are called as the independent elements of real symmetric matrix $X=\left(x_{\mathrm{ij}}\right) \in \mathrm{SR}^{n \times n}$, for short independent entry. Denoted by

$\operatorname{vec}_{S}(X)=\left(\alpha_{1}, \alpha_{2}, \ldots \alpha_{n}\right)^{T} \in R^{\frac{n(n+1)}{2}}$

is called a column straight of the independent elements of real symmetric matrix $X=\left(x_{\mathrm{ij}}\right) \in \mathrm{SR}^{n \times n}$.

Definition $4^{[1]}$ For arbitrary real dissymmetric matrix $X=\left(x_{\mathrm{ij}}\right) \in \mathrm{ASR}^{n \times n}$, and let $\beta_{1}=\left(x_{21}, x_{31}, \ldots x_{n 1}\right), \beta_{2}=\left(x_{32}, x_{42}, \ldots x_{n 2}\right), \ldots, \beta_{n-1}=\left(x_{(n-1)(n-2)}, x_{n(n-2)}\right), \beta_{n}=x_{n(n-1)}$, the elements of $\beta_{1}, \beta_{2}, \ldots, \beta_{n}$ are called as the independent elements of real dissymmetric matrix dissymmetric $\operatorname{matrix} X=\left(x_{\mathrm{ij}}\right) \in \mathrm{ASR}^{n \times n}$. Denoted by

$$
\operatorname{ves}_{A}(X)=\left(\beta_{1}, \beta_{2}, \ldots \beta_{n}\right)^{T} \in R^{\frac{n(n-1)}{2}}
$$

is called a column straight of the independent elements of real dissymmetric matrix

Lemma $5^{[1]}$ For $X=\left(x_{\mathrm{ij}}\right) \in \mathrm{SR}^{n \times n}$. Then

$X \in \mathrm{SR}^{n \times n} \Leftrightarrow \operatorname{ves}(X)=K_{n} \operatorname{ves}_{S}(X), K_{n} \in R^{n^{2} \times \frac{n(n+1)}{2}}$,

\begin{tabular}{|c|c|c|c|c|}
\hline$e_{1}$ & $a m p ; e_{2}$ & $a m p ; e_{3}$ & $a m p ; \cdots$ & $a m p ; e_{n-1}$ \\
\hline 0 & $a m p ; e_{1}$ & $a m p ; 0$ & $a m p ; \cdots$ & $a m p ; 0$ \\
\hline 0 & 0 & $e_{1}$ & amp; & amp; 0 \\
\hline & $a m p ; 0$ & $a m p$ & $a m p ; \cdots$ & $a m p ; e_{1}$ \\
\hline & 0 & 0 & $a m p ; \cdots$ & $a m p ; 0$ \\
\hline
\end{tabular}

in which $K_{n}=($ 


\begin{tabular}{|c|c|c|c|c|}
\hline$e_{n}$ & amp; 0 & $a m p ; 0$ & amp; $\cdots$ & amp; 0 \\
\hline 0 & $a m p ; e_{2}$ & $a m p ; e_{3}$ & $a m p ; \cdots$ & $a m p ; e_{n-1}$ \\
\hline 0 & 0 & 0 & & 0 \\
\hline$\cdots$ & $\cdots$ & $\ldots$ & amp & amp; . \\
\hline 0 & $a m p ; 0$ & $a m p$ & $a m p ; \cdots$ & $a m p ; e_{2}$ \\
\hline$e_{1}$ & 0 & 0 & $a m p ; \cdots$ & amp; 0 \\
\hline 0 & amp; $\cdots$ & $a m p ; 0$ & $0 \quad a m p ; 0$ & amp; 0 \\
\hline$e_{n}$ & amp; $\cdots$ & $a m p ; 0$ & amp; 0 & amp; 0 \\
\hline 0 & $\ldots$ & $\ldots$ & $a m p ; \ldots$ & $a m p ; \stackrel{0}{\ldots}$ \\
\hline 0 & $a m p ; \ldots$ & $a m p$ & $a m p ; e_{n}$ & $a m p ; 0$ \\
\hline$e_{2}$ & $\ldots$ & 0 & $a m p ; e_{n-1}$ & $a m p ; e_{n}$ \\
\hline
\end{tabular}

Lemma $6^{[1]} \operatorname{For} X=\left(x_{\mathrm{ij}}\right) \in R^{n \times n}$. Then

$X \in \mathrm{ASR}^{n \times n} \Leftrightarrow \operatorname{ves}(X)=L_{n} \operatorname{ves}_{A}(X), L_{n} \in R^{n^{2} \times \frac{n(n+1)}{2}}$,

in which $L_{n}=($

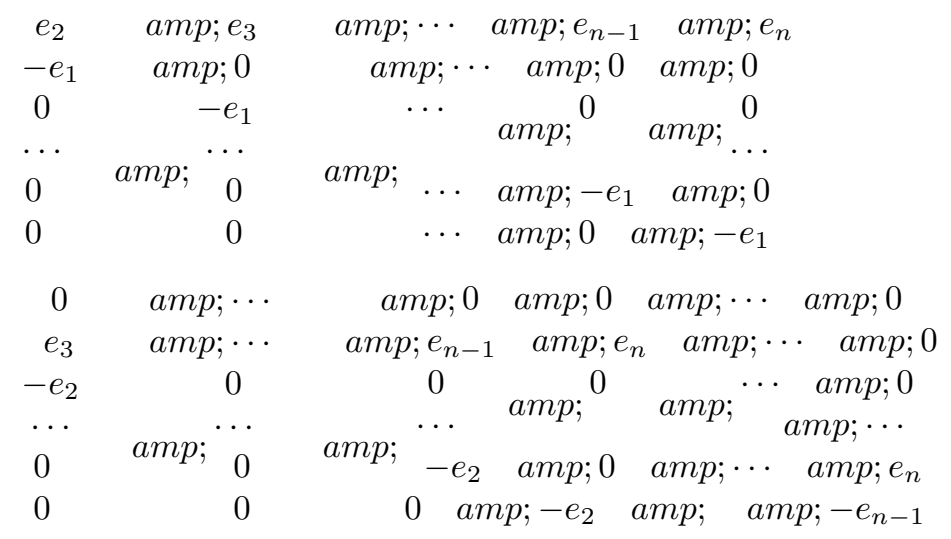

Lemma $7^{[1]}$ Let $A \in R^{m \times n}, b \in R^{n}$. Then the least square solutions of the incompatible linear equations $A x=b$ is $x=A^{+} b+\left(I-A A^{+}\right) y$, where $y \in R^{n}$ is arbitrary vector.

2 The minimum norm least square solution of complex matrix equations $(\mathrm{AXB}, \mathrm{CXD})=(\mathrm{E}, \mathrm{F})$

In this paper, the special structure of the real representation matrix of the complex matrix and the MP inverse property of the matrix, we study Hermitian least square solutions of complex matrix equations(1), and give the expressions of Hermitian minimum norm least square solution, real symmetric minimum norm least square solution and real dissymmetric minimum norm least square solution.

Theorem 1 Let $A, C \in C^{m \times n}, B, D \in C^{n \times s}, E, F \in C^{m \times s}, X=X_{0}+X_{1} i$,where $X_{0} \in \mathrm{SR}^{n \times n}, X_{1} \in$ $\mathrm{ASR}^{n \times n}$. For complex matrix equations (1), the Hermitian minimum norm least square solution $X$ satisfies

$\left(\frac{\operatorname{ves}_{S}\left(X_{0}\right)}{\left(\operatorname{ves}_{A}\left(X_{1}\right)\right)}\right)=\left[\left(\frac{\left(B_{c}^{R}\right)^{T} \otimes A^{R}}{\left.\left(D_{c}^{R}\right)^{T} \otimes C^{R}\right)}\right) \mathrm{HVK} K_{n}\right]^{+}\left(\frac{E_{c}^{R}}{F_{c}^{R}}\right)$.

Proof. By lemma 1-6, we get

$$
\|\mathrm{AXB}-E\|+\|\mathrm{CXD}-F\|
$$




$$
\begin{gathered}
=\left\|A^{R} \mathrm{X}^{R} B_{c}^{R}-E_{c}^{R}\right\|+\left\|C^{R} \mathrm{X}^{R} D_{c}^{R}-F_{c}^{R}\right\| \\
=\left\|\left(\left(B_{c}^{R}\right)^{T} \otimes A^{R}\right) \operatorname{vec}\left(\mathrm{X}^{R}\right)-\operatorname{vec}\left(E_{c}^{R}\right)\right\|+\left\|\left(\left(D_{c}^{R}\right)^{T} \otimes C^{R}\right) \operatorname{vec}\left(\mathrm{X}^{R}\right)-\operatorname{vec}\left(F_{c}^{R}\right)\right\| \\
=\left\|\left(\frac{\left(\left(B_{c}^{R}\right)^{T} \otimes A^{R}\right) \operatorname{vec}\left(X^{R}\right)-\operatorname{vec}\left(E_{c}^{R}\right)}{\left(\left(D_{c}^{R}\right)^{T} \otimes C^{R}\right) \operatorname{vec}\left(X^{R}\right)-\operatorname{vec}\left(F_{c}^{R}\right)}\right)\right\| \\
=\left\|\left(\frac{\left(\left(B_{c}^{R}\right)^{T} \otimes A^{R}\right)}{\left(\left(D_{c}^{R}\right)^{T} \otimes C^{R}\right)}\right) \operatorname{vec}\left(X^{R}\right)-\left(\frac{E_{c}^{R}}{F_{c}^{R}}\right)\right\| \\
=\left\|\left(\frac{\left(\left(B_{c}^{R}\right)^{T} \otimes A^{R}\right)}{\left(\left(D_{c}^{R}\right)^{T} \otimes C^{R}\right)}\right) \operatorname{HK}\left(\begin{array}{c}
\operatorname{vec} X_{0} \\
\operatorname{vec} X_{1}
\end{array}\right)-\left(\frac{E_{c}^{R}}{F_{c}^{R}}\right)\right\| \\
=\left\|\left(\frac{\left(\left(B_{c}^{R}\right)^{T} \otimes A^{R}\right)}{\left(\left(D_{c}^{R}\right)^{T} \otimes C^{R}\right)}\right) \operatorname{HK}\left(\begin{array}{l}
K_{n} \operatorname{ves}_{S}\left(X_{0}\right) \\
L_{n} \operatorname{ves}_{A}\left(X_{1}\right)
\end{array}\right)-\left(\frac{E_{c}^{R}}{F_{c}^{R}}\right)\right\|
\end{gathered}
$$

Where $H, K, K_{n}, L_{n}$ are defined in the form of lemma 4-6. Let $G=\operatorname{diag}\left(K_{n}, L_{n}\right)$, then

$$
\begin{aligned}
& \|\mathrm{AXB}-E\|+\|\mathrm{CXD}-F\|=\|\left(\frac{\left(\left(B_{c}^{R}\right)^{T} \otimes A^{R}\right)}{\left(\left(D_{c}^{R}\right)^{T} \otimes C^{R}\right)}\right) \text { HKG } \\
& \left(\begin{array}{c}
\operatorname{ves}_{S}\left(X_{0}\right) \\
\operatorname{ves}_{A}\left(X_{1}\right)
\end{array}\right)-\left(\frac{E_{c}^{R}}{F_{c}^{R}}\right),
\end{aligned}
$$

So calculating the least squares solution of the complex matrix equation (1) is equivalent to calculating the least squares solution of the following real linear system

$$
\begin{aligned}
& \left(\frac{\left(\left(B_{c}^{R}\right)^{T} \otimes A^{R}\right)}{\left(\left(D_{c}^{R}\right)^{T} \otimes C^{R}\right)}\right) \text { HKG } \\
& \left(\begin{array}{c}
\operatorname{ves}_{S}\left(X_{0}\right) \\
\operatorname{ves}_{A}\left(X_{1}\right)
\end{array}\right)=\left(\frac{E_{c}^{R}}{F_{c}^{R}}\right) .
\end{aligned}
$$

By lemma 7, the set of Hermitian least square solution of (1) is denoted by

$C_{H}=\{X \mid$

$$
\left(\begin{array}{c}
\operatorname{ves}_{S}\left(X_{0}\right) \\
\operatorname{ves}_{A}\left(X_{1}\right)
\end{array}\right)=\left[\left(\frac{\left(\left(B_{c}^{R}\right)^{T} \otimes A^{R}\right)}{\left(\left(D_{c}^{R}\right)^{T} \otimes C^{R}\right)}\right) H K G\right]^{+}+\left[\left(I-\left[\left(\frac{\left(\left(B_{c}^{R}\right)^{T} \otimes A^{R}\right)}{\left(\left(D_{c}^{R}\right)^{T} \otimes C^{R}\right)}\right) H K G\right]^{+}\left(\frac{\left(\left(B_{c}^{R}\right)^{T} \otimes A^{R}\right)}{\left(\left(D_{c}^{R}\right)^{T} \otimes C^{R}\right)}\right) \text { HKG }\right] y\right\},
$$

Where $y$ is arbitrary vector. In particular, the Hermitian minimum norm least square solution of (1) is can be expressed as $X=X_{0}+X_{1} i \in C^{n \times n}$, in which $X_{0} X_{1}$ satisfies

$$
\left(\begin{array}{c}
\operatorname{ves}_{S}\left(X_{0}\right) \\
\operatorname{ves}_{A}\left(X_{1}\right)
\end{array}\right)=\left[\left(\frac{\left(B_{c}^{R}\right)^{T} \otimes A^{R}}{\left.\left(D_{c}^{R}\right)^{T} \otimes C^{R}\right)}\right) H K G\right]^{+}\left(\frac{E_{c}^{R}}{F_{c}^{R}}\right) \text { 证毕. }
$$

Corollary 1 Let $A, C \in C^{m \times n}, B, D \in C^{n \times s}, E, F \in C^{m \times s}, X=X_{0}+X_{1} i$, in which $X_{1}=0, X_{0} \in \mathrm{SR}^{n \times n}$. For complex matrix equations (1), the real symmetric minimum norm least square solution $X=X_{0}$ satisfies 
$\operatorname{ves}_{S}\left(X_{0}\right)=\left[\left(\frac{\left(B_{c}^{R}\right)^{T} \otimes A^{R}}{\left.\left(D_{c}^{R}\right)^{T} \otimes C^{R}\right)}\right) \operatorname{HV} K_{n}\right]^{+}\left(\frac{E_{c}^{R}}{F_{c}^{R}}\right)$,

Where $H, V, \mathrm{~K}_{n}$ are defined in the form of lemma 4-6.

Corollary 2Let $A, C \in C^{m \times n}, B, D \in C^{n \times s}, E, F \in C^{m \times s}, X=X_{0}+X_{1} i$,in which $X_{1}=0, X_{0} \in \mathrm{ASR}^{n \times n}$. For complex matrix equations (1), the real dissymmetric minimum norm least square solution $X=X_{0}$ satisfies

$\operatorname{ves}_{A}\left(X_{0}\right)=\left[\left(\frac{\left(B_{c}^{R}\right)^{T} \otimes A^{R}}{\left.\left(D_{c}^{R}\right)^{T} \otimes C^{R}\right)}\right) \operatorname{HV} L_{n}\right]^{+}\left(\frac{E_{c}^{R}}{F_{c}^{R}}\right)$,

Where $H, V, \mathrm{~L}_{n}$ are defined in the form of lemma 4-6.

\section{Algorithm}

For complex matrix equations (1), in which $A, C \in C^{m \times n}, B, D \in C^{n \times s}, E, F \in C^{m \times s}$. the following Theorem 1, Corollary 1 and Corollary 2, we propose the following algorithms for solving the Hermitian minimum norm least square solution, the real symmetric minimum norm least square solution and the real dissymmetric minimum norm least square solution of (1).

Algorithm 1(solving the Hermitian minimum norm least square solution of (1) )

(1) Input $A, B, C, D, E, F H, K, G$,

(2) Form $\mathrm{A}^{R}, C^{R}, \mathrm{~B}_{c}^{R}, D_{c}^{R}, E_{c}^{R}, \mathrm{~F}_{c}^{R}$,

(3) Calculat

$\left(\begin{array}{c}\operatorname{ves}_{S}\left(X_{0}\right) \\ \operatorname{ves}_{A}\left(X_{1}\right)\end{array}\right)=\left[\left(\frac{\left(B_{c}^{R}\right)^{T} \otimes A^{R}}{\left.\left(D_{c}^{R}\right)^{T} \otimes C^{R}\right)}\right) H K G\right]^{+}\left(\frac{E_{c}^{R}}{F_{c}^{R}}\right)$.

Algorithm 2 (solving the real symmetric minimum norm least square solution of (1))

(1) Input $A, B, C, D, E, F H, V, K_{n}$,

(2) Form $A^{R}, C^{R}, B_{c}^{R}, D_{c}^{R}, E_{c}^{R}, F_{c}^{R}$,

(3) Calculat $\operatorname{ves}_{S}\left(X_{0}\right)=\left[\left(\frac{\left(B_{c}^{R}\right)^{T} \otimes A^{R}}{\left.\left(D_{c}^{R}\right)^{T} \otimes C^{R}\right)}\right) \mathrm{HV}_{n}\right]^{+}\left(\frac{E_{c}^{R}}{F_{c}^{R}}\right)$.

Algorithm 3 (solving the real dissymmetric minimum norm least square solution of (1))

(1) Input $A, B, C, D, E, F H, V, L_{n}$,

(2) Form $A^{R}, C^{R}, B_{c}^{R}, D_{c}^{R}, E_{c}^{R}, F_{c}^{R}$,

(3) Calculat $\operatorname{ves}_{A}\left(X_{0}\right)=\left[\left(\frac{\left(B_{c}^{R}\right)^{T} \otimes A^{R}}{\left.\left(D_{c}^{R}\right)^{T} \otimes C^{R}\right)}\right) \mathrm{HV}_{n}\right]^{+}\left(\frac{E_{c}^{R}}{F_{c}^{R}}\right)$.

\section{Conclusious}

In this paper, by using the special structures of the real representation of complex matrix, the vec operator of the matrix, the Kronecker product of matrices and the properties of MP inverse of matrix, we transform the calculation problem of the special least squares solutions of complex matrix equations into the least squares problem of corresponding linear systems, and propose illustrate the effectiveness based on real matrix with the Hermitian minimum norm least square solution, the real symmetric minimum norm least square solution and the real dissymmetric minimum norm least square solution of the complex matrix equation $(A X B, C X D)=(E, F)$.

\section{References}


[1] Zhang Fengxia, Wei Musheng, Li Ying, et al. The minimal norm least squares Hermitian solution of the complex matrix equation $A X B+C X D=E[\mathrm{~J}]$. Journal of the Franklin Institute, 2018, 355(3): 1296-1310.

[2] Zhang Fengxia, Wei Musheng, Li Ying, et al. An efficient method for special least squares solution of the complex matrix equation $(A X B, C X D)=(E, F)[\mathrm{J}]$. Journal of the Franklin Institute, 2018, 76(8): 2001-2010.

[3] Wang Peng, Yuan Shifang, Xie Xianyun. Least squares Hermitian problem of complex matrix equation $(A X B, C X D)=(E, F)[\mathrm{J}]$. Journal of Inequalities and Applications, 2016, 2016(1): 296.

[4] Yuan Shifang, Liao Anping. Least squares Hermitian solution of the complex matix equation $A X B+$ $C X D=E$ with least norm[J]. Journal of the Franklin Institute, 2014, 351: 4978-4997.

[5] Yuan Shifang. The symmetric minimal norm squares solution of matrix equation $Z X B+C Y D=E[J]$. Journal of hunan university, 2007, 29(2): 203-216.

[6] Yuan Shifang, Liao Anping. Anticentral symmetric solution and its excellent approximation of matrix equation $(A X, X B)=(C, D)[\mathrm{J}]$. Journal of mathematical theory and application, 2005, 24(1): 86-90.

[7] Yao Guozhu, Liao Anping, Duan Xuefeng. The minimal square Hamilton solution of matrix equation $A X B=C[J]$. Journal on Numerical Methods and Computer Application, 2009, 30(1): 48-57.

[8] Yao Guozhu. The anti-centrosymmetric solution of $A X B=C$ on a linear manifold. Journal of Changsha university of science and technology, 2004, 2004(3): 78-83.

[9] Peng Zhuohua, Hu Xiyan, Zhang Lei. The least squares bisymmetric solution of a class of matrix equations and its best approximation, Journal of hunan university, 2007, 34(9): 78-81.

[10] Li Ying, Wei Musheng, Zhang Fangxia, et al. Real Structure-preserving Algorithms for the Quaternion Cholesky Decomposition: Revisit. Journal of Liaocheng University, 2018, 32(1): 27-34.

[11] Song Caiqin, Chen Guoliang. Solution to matrix equations $X-A X B=C Y+\operatorname{Rand} X-A X B=$ $C Y+R[\mathrm{~J}]$. Journal of computational and Applied Mathematics, 2018, 343(1): 488-500.

[12] Dai Hua. Matrix theory[M]. Beijing, Beijing Science Press, 2001: 233-248. 